\title{
AbobotulinumtoxinA using 2-mL dilution (500 U/2-mL) maintains durable improvement across multiple treatment cycles
}

Khashayar Dashtipour ${ }^{1 *}$, Stefan Wietek ${ }^{2}$, Bruce Rubin ${ }^{3}$, Pascal Maisonobe ${ }^{4}$, Laxman Bahroo ${ }^{5}$ and Richard Trosch $^{6}$

\begin{abstract}
Background: Cervical dystonia (CD), the most common focal dystonia, is a chronic neurological movement disorder characterized by sustained involuntary contractions of the neck muscles, leading to abnormal postures. AbobotulinumtoxinA (aboBoNT-A) was approved in the US initially as a $500 \mathrm{U}$ per 1-mL dilution and subsequently, as a $500 \mathrm{U} / 2-\mathrm{mL}$ dilution (or $250 \mathrm{U} / \mathrm{mL}$ ), thereby providing clinicians with more flexible dosing options to better meet individual patient needs. The objective of this open-label extension study was to evaluate the longer term safety and efficacy of repeat treatments with aboBoNT-A using 2-mL dilutions in adults with cervical dystonia.

Methods: Patients ( $N=112$ ) from a 12-week, double-blind lead-in study (NCT01753310) received up to three additional treatments of aboBoNT-A, with re-treatment every 12-16 weeks based on clinical judgment. Safety was assessed through treatment-emergent adverse events (TEAEs). The Toronto Western Spasmodic Torticollis Rating Scale (TWSTRS) total and subscale scores were measured at day 1 of each treatment cycle (C), 4 weeks after each treatment, and 12 weeks after the third treatment. Descriptive statistics were used for all analyses.

Results: In cycles 1, 2, 3, and 4, respectively, 35.7, 25.9, 30.2, and 22.8\% of patients reported TEAEs. Dysphagia, muscular weakness, and neck pain were each reported by $10.7 \%$ of patients, over the full study duration. Mean TWSTRS total score decreased from 37.7 (SD 13.6 [C1, day 1]) to 30.1 (SD 12.8 [C3, week 12]). In each cycle, TWSTRS total and subscale scores decreased from day 1 to week 4 and increased between weeks 4 and 12, though the week 12 scores remained lower than day 1 scores.
\end{abstract}

Conclusion: Extended treatment of cervical dystonia with aboBoNT-A (up to 3 additional treatment cycles) using a 2-mL dilution is effective, with a positive risk-benefit profile.

Trial registration: ClinicalTrials.gov Identifier: NCT01753336. Registered 17 Dec 2012.

Keywords: AbobotulinumtoxinA, Safety, Efficacy, Cervical dystonia, Open-label extension study, Repeat treatments

\section{Introduction}

Cervical dystonia (CD), the most common focal dystonia, is a chronic neurological movement disorder characterized by sustained involuntary contractions of the

\footnotetext{
* Correspondence: kdashtipour@llu.edu

'Loma Linda University School of Medicine, Loma Linda, CA 92350, USA

Full list of author information is available at the end of the article
}

neck muscles, leading to a disabling posture [1]. The epidemiology of CD is not well characterized due to highly variable study designs and study populations [2]. However, a review of insurance records for a racially diverse population in California found the minimum estimated overall incidence of $\mathrm{CD}$ to be 8 per million person-years, with the preponderance of cases occurring among whites

(c) The Author(s). 2020 Open Access This article is licensed under a Creative Commons Attribution 4.0 International License, which permits use, sharing, adaptation, distribution and reproduction in any medium or format, as long as you give appropriate credit to the original author(s) and the source, provide a link to the Creative Commons licence, and indicate if changes were made. The images or other third party material in this article are included in the article's Creative Commons licence, unless indicated otherwise in a credit line to the material. If material is not included in the article's Creative Commons licence and your intended use is not permitted by statutory regulation or exceeds the permitted use, you will need to obtain permission directly from the copyright holder. To view a copy of this licence, visit http://creativecommons.org/licenses/by/4.0/ The Creative Commons Public Domain Dedication waiver (http://creativecommons.org/publicdomain/zero/1.0/) applies to the data made available in this article, unless otherwise stated in a credit line to the data. 
versus other races (12.3 vs. 1.5 per million person-years) [3]. Moreover, women were twice as likely as men to be diagnosed with $\mathrm{CD}$. Although the number of affected patients is relatively small, with few treatment options, the disabling impact of this disorder can be great for patients and their families.

AbobotulinumtoxinA, one of four currently approved botulinum toxin preparations for treatment of $\mathrm{CD}$, received the indication using a $500 \mathrm{U} / 1-\mathrm{mL}$ dilution in the United States of America in 2009. Approval was based on two pivotal studies $[4,5]$ and their respective openlabel extension studies [6]. To provide clinicians with more flexible dosing to better meet individual patient needs, the safety and efficacy of a $500 \mathrm{U} / 2-\mathrm{mL}$ dilution (or $250 \mathrm{U} / \mathrm{mL}$ ) was investigated. The subsequent approval of the 2-mL dilution was based on results of a 12-week, phase 3b, multicenter, randomized, doubleblind, placebo-controlled trial in adults with $C D$ (NCT01753310) [7]. This study demonstrated that abobotulinumtoxinA significantly improved symptoms in both toxin-naïve and previously treated patients, with a safety profile consistent with the 1-mL dilution. In this open-label extension (OLE) of the 12-week, double-blind study (NCT01753336), we assess the longer term safety and efficacy of abobotulinumtoxin A using a 2-mL dilution in adults with $\mathrm{CD}$ for up to 3 additional treatment cycles.

\section{Methods}

\section{Study design}

This was a phase $3 \mathrm{~b}$, prospective, multicenter, OLE study to a double-blind, placebo-controlled lead-in study (NCT01753310) [7] that evaluated the efficacy and safety of a single cycle of abobotulinumtoxinA using a 2-mL dilution in adults with $\mathrm{CD}$. The current study incorporated a maximum of three additional treatment cycles, occurring at intervals of no less than 12 weeks, a followup visit 4 weeks after each treatment, and an end-ofstudy visit 12 weeks after the third treatment visit (Fig. 1). Patients in the lead-in placebo group had exposure of up to three cycles, whereas those in the lead-in abobotulinumtoxinA group had overall exposure of up to four treatment cycles.

The OLE study was conducted under the provisions of the Declaration of Helsinki and in accordance with the International Conference on Harmonisation (ICH) Consolidated Guideline on Good Clinical Practice. In addition, this study adhered to relevant company policies and all U.S. Food and Drug Administration (FDA) regulatory requirements. Study protocol and amendments were approved by an independent Institutional Review Board and all patients or their legal representative signed an informed consent form.

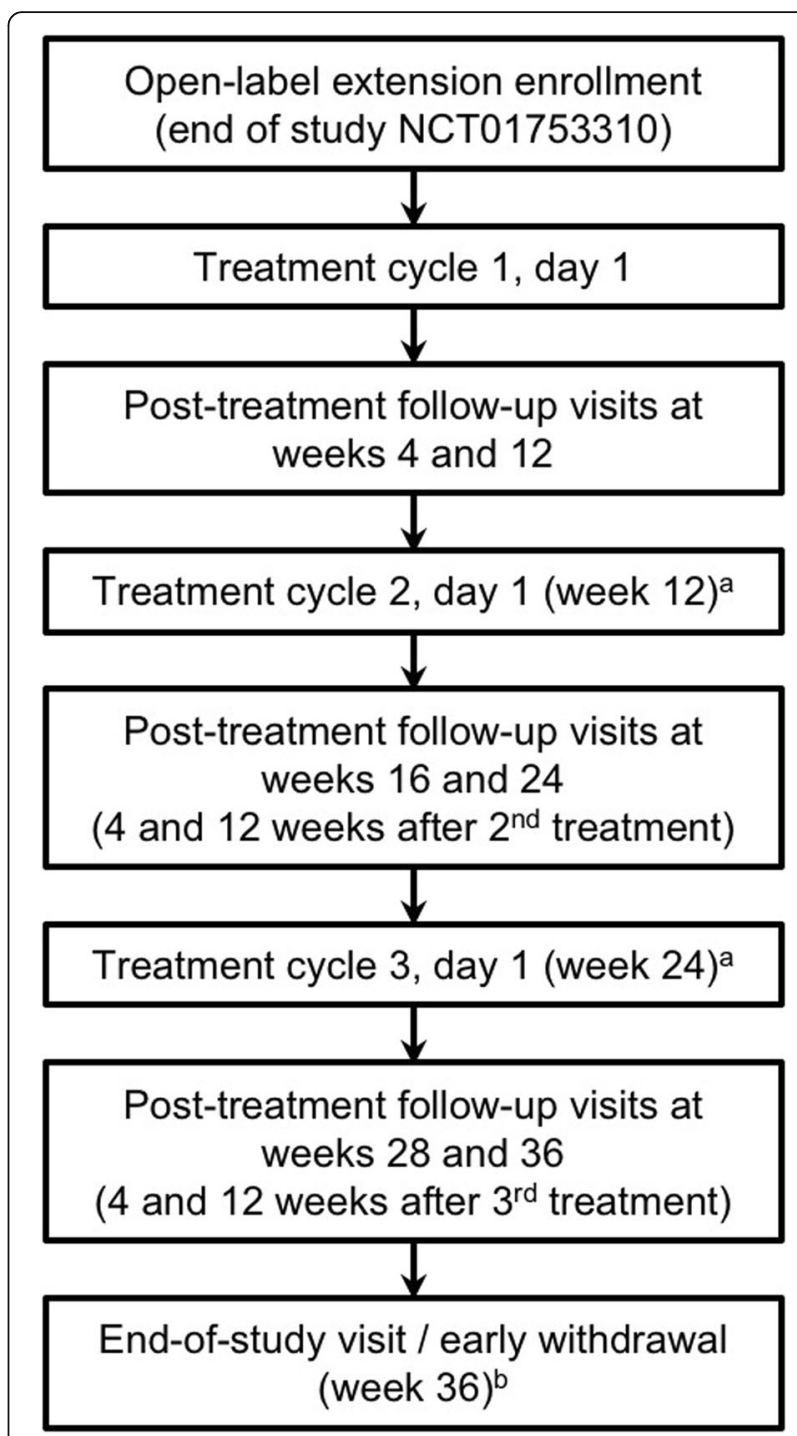

Fig. 1 Study Flow Chart. ${ }^{\text {TThis }}$ visit should occur on the same day as the last visit of the previous treatment cycle. Re-treatment was expected to occur approximately 12-16 weeks after the last treatment; however, the exact timing of treatment was determined by the investigator based on clinical need. 'Patients who withdrew from the study early (ie, before week 12 of cycle 3 ) underwent all procedures required for the week 36 visit

Adult patients who completed the week 12 visit of the lead-in study, or patients who withdrew after week 4 and whose TWSTRS [8] total score between weeks 4 and 8 was reduced by $\leq 15 \%$ from baseline, were invited to participate in this OLE study.

\section{Treatment}

The OLE incorporated up to three additional treatment cycles, with re-treatment occurring every 12 to 16 weeks based on clinical judgment. Day 1, OLE cycle 1 was the same as the last lead-in study visit. For OLE cycle 1, patients from the placebo group at lead-in baseline 
received abobotulinumtoxinA $500 \mathrm{U} / 2 \mathrm{~mL}$ in $\geq 2$ affected neck muscles and patients from the treatment group at lead-in baseline received the same starting dose of abobotulinumtoxinA dose $(250-500 \mathrm{U} / 2 \mathrm{~mL})$ received in the lead-in study injected into previously treated muscles. For cycles 2 and 3, the need for dose adjustments was determined by the investigator, with allowed increments and decrements limited to $\leq 250 \mathrm{U} /$ cycle and maximal total dose limited to $1000 \mathrm{U} /$ cycle.

\section{Safety and tolerability}

Safety and tolerability were assessed by recording TEAEs, as well as through physical examinations, vital signs, and clinical laboratory assessments. These assessments were performed on day 1 of each treatment cycle, which occurred 12 to 16 weeks apart, and at the last study visit or early withdrawal visit.

\section{Efficacy}

Efficacy assessments were based on TWSTRS total and subscale (severity, disability, and pain) scores. Scores could range from 0 to 35,0 to 30 , and 0 to 20 , for the severity, disability, and pain subscales, respectively. TWSTRS total scores range from 0 (best) to 85 (worst) and comprise the sum of the three individual scores' subscales. TWSTRS assessments were conducted by the investigator prior to the administration of study treatment at baseline (day 1 of treatment cycle 1) and at posttreatment visits.

\section{Statistical analyses}

All analyses are descriptive in nature and were conducted using the safety population, which included all patients who received $\geq 1$ dose of study treatment and had $\geq 1$ safety record post treatment. Adverse events were assigned to a specific cycle based on the first exposure to abobotulinumtoxinA; therefore, patients who received abobotulinumtoxin $\mathrm{A}$ in the lead-in study had four cycles, whereas patients who received placebo in the lead-in study had three cycles. Statistical analyses of efficacy and safety data were performed using Statistical Analysis System (SAS) (version 9.4).

\section{Results}

\section{Patients and dosing}

A total of 112 patients were enrolled and treated, and 92 (82.1\%) completed the week 36 visit (cycle 3, week 12) (Fig. 2). No patients were excluded from the safety analysis set; 20 patients (17.9\%) withdrew from the study due to personal reasons $(n=12)$, lost-to-follow-up $(n=3)$, investigator's decision $(n=2)$, sponsor's decision $(n=2)$, or other $(n=1)$. No patients withdrew due to an adverse event. Cycle durations lasted approximately 13 weeks (Cycle 1, median 12.0 [range 3-29]; Cycle 2, median 12.0 [range 8-15]; Cycle 3, median 13.0 [range 8-58).

Of the 112 patients enrolled, 77 (69\%) had prior neurotoxin exposure (non-naïve), and 35 (31\%) had no neurotoxin exposure prior to entering the lead-in study (toxin-naïve). Patients' median age was 56 (range 29-82) years, 94.6\% were white, and 62.5\% were female (Table 1). The mean body mass index was $27.0( \pm 4.9) \mathrm{kg} / \mathrm{m}^{2}$.

The total dose and volume of abobotulinumtoxinA administered by treatment cycle, with overall results, are summarized in Table 2. The median dose administered

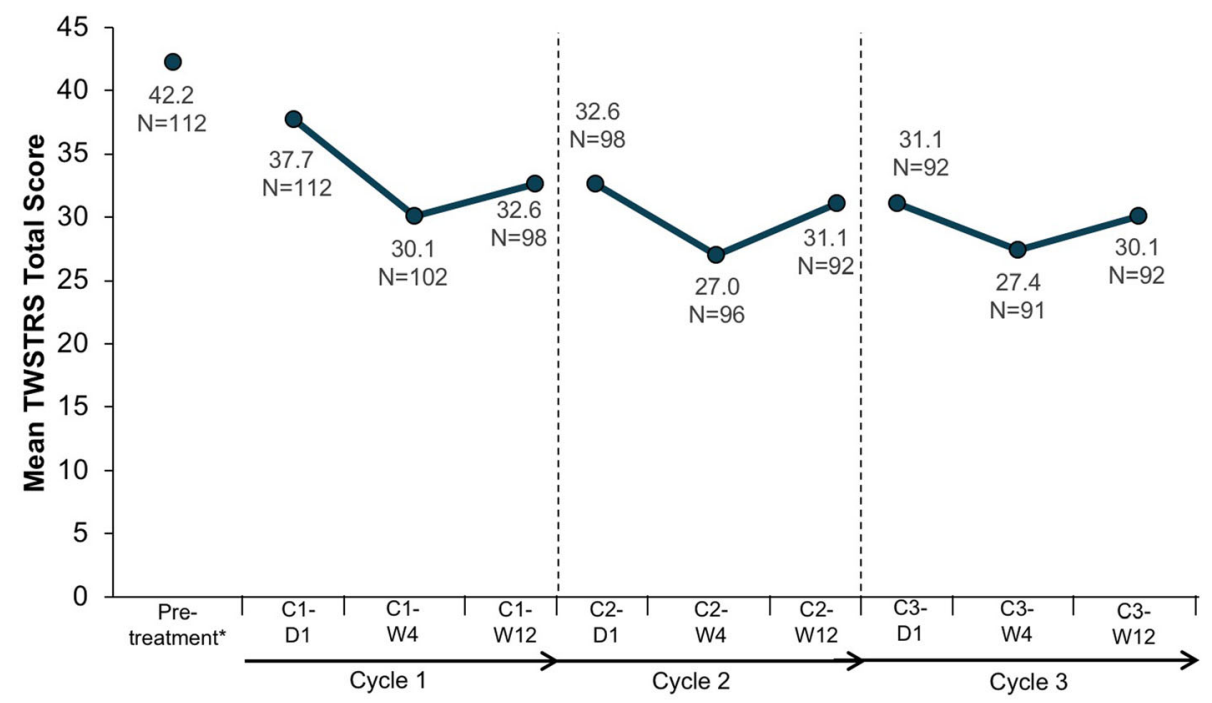

Fig. 2 Change from baseline in TWSTRS total score. C, cycle; D, day; W, week. *Before first abobotulinumtoxinA administration 
Table 1 Demographics and baseline characteristics

\begin{tabular}{ll}
\hline & Patients $(N=112)$ \\
\hline Toxin-naïve & $35(31.3 \%)$ \\
Non-naive & $77(68.8 \%)$ \\
Age, years & \\
Mean & $57.2(11.0)$ \\
Median (range) & $56(29,82)$ \\
Sex, $n$ (\%) & \\
Male & $42(37.5 \%)$ \\
Female & $70(62.5 \%)$ \\
BMl, kg/m ${ }^{2}$ & \\
Mean (SD) & $27.0(4.9)$ \\
Median & 26 \\
Range & $18-46$ \\
Race, $n$ (\%) & \\
White & $106(94.6 \%)$ \\
Black & $2(1.8 \%)$ \\
Asian & $3(2.7 \%)$ \\
Multiple & $1(0.9 \%)$ \\
Ethnicity, $n$ (\%) & \\
Hispanic or Latino & $16(14.3 \%)$ \\
Not Hispanic or Latino & $96(85.7 \%)$ \\
\hline
\end{tabular}

by treatment cycle was $500 \mathrm{U}$ and ranged from $200 \mathrm{U}$ to $500 \mathrm{U}$ in cycle $1 ; 150 \mathrm{U}$ to $806 \mathrm{U}$ in cycle 2 ; and $163 \mathrm{U}$ to $1000 \mathrm{U}$ in cycle 3 in all patients. The mean total dose increased over treatment cycles, with toxin-naive patients receiving higher doses on average than did non-naïve patients. Median dose for toxin-naïve patients was 1500 $\mathrm{U}$, ranging from $500 \mathrm{U}$ to $2250 \mathrm{U}$, and $1444 \mathrm{U}$ in nonnaïve patients, ranging from $263 \mathrm{U}$ to $2031 \mathrm{U}$. The mean dose in the OLE third cycle was $596.5 \mathrm{U}$ (SD 192.4) for toxin-naïve patients and 494.5 U (SD 143.4) among patients with prior exposure. A median of five muscles were injected at five sites for all patients and non-naïve patients during all treatment cycles and for naïve patients at treatment cycle 1 . At treatment cycles 2 and 3 , naive patients had injections in a median of six muscles at six sites. The most frequently injected muscle was the splenius capitis for $88.4,89.7$, and $90.3 \%$ of patients during treatment cycles 1,2 , and 3 , respectively (Table 3 ). More than $50 \%$ of patients had injections in their sternocleidomastoid, trapezius, and levator scapulae muscles in each treatment cycle.

\section{Safety and tolerability}

A total of 220 TEAEs were reported among 70 patients (62.5\%) (Table 4). The percentages of patients reporting TEAEs in cycles $1,2,3$, and 4 were $35.7,25.9,30.2$, and $22.8 \%$, respectively. Dysphagia, muscular weakness, and neck pain were the most frequent TEAEs (10.7\% each). Of the 12 patients who reported dysphagia; 6 patients received injections into the sternocleidomastoid muscle with doses ranging from $50 \mathrm{U}$ to $250 \mathrm{U}, 3$ patients received no injections into the sternocleidomastoid muscle, and 1 patient had 2 incidences of dysphagia, once after receiving a $75 \mathrm{U}$ injection and once without an injection into the sternocleidomastoid muscle. Details regarding muscles injected were unavailable for 2

Table 2 AbobotulinumtoxinA administration by treatment cycle

\begin{tabular}{|c|c|c|c|c|}
\hline & Treatment Cycle 1 & Treatment Cycle 2 & Treatment Cycle 3 & All Treatment Cycles \\
\hline All patients, $n$ & 112 & 97 & 93 & 112 \\
\hline Total dose, administered in Units, mean (SD) & $457.7(75.3)$ & $507.1(130.3)$ & $527.4(166.8)$ & $1334.8(476.9)$ \\
\hline Total dose <500 U, n (\%) & $38(33.9 \%)$ & $31(32.0 \%)$ & $30(32.3 \%)$ & - \\
\hline Total dose $=500 \cup, n(\%)$ & $74(66.1 \%)$ & $41(42.3 \%)$ & $38(40.9 \%)$ & - \\
\hline Total dose > 500 U, n (\%) & 0 & $25(25.8 \%)$ & $25(26.9 \%)$ & - \\
\hline Toxin-naïve patients, $n$ & 35 & 31 & 30 & 35 \\
\hline Total dose, administered in Units, mean (SD) & $492.9(34.2)$ & $549.5(114.6)$ & $596.5(192.4)$ & $1490.9(468.6)$ \\
\hline Total dose <500 U, n (\%) & $2(5.7 \%)$ & $5(16.1 \%)$ & $5(16.7 \%)$ & - \\
\hline Total dose $=500 \cup, n(\%)$ & $33(94.3 \%)$ & $15(48.4 \%)$ & $15(50.0 \%)$ & - \\
\hline Total dose > 500 U, n (\%) & 0 & $11(35.5 \%)$ & $10(33.3 \%)$ & - \\
\hline Non-naïve patients, $n$ & 77 & 66 & 63 & 77 \\
\hline Total dose, administered in Units, mean (SD) & $441.7(83.2)$ & $487.1(133.2)$ & $494.5(143.4)$ & $1263.8(466.5)$ \\
\hline Total dose <500 U, n (\%) & $36(46.8 \%)$ & $26(39.4 \%)$ & $25(39.7 \%)$ & - \\
\hline Total dose $=500 \cup, n(\%)$ & $41(53.2 \%)$ & $26(39.4 \%)$ & $23(36.5 \%)$ & - \\
\hline Total dose > $500 \cup, n(\%)$ & 0 & $14(21.2 \%)$ & $15(23.8 \%)$ & - \\
\hline
\end{tabular}

Drug exposure for each cycle was calculated as the sum of all doses within a cycle, across all injection sites. Total cumulative drug exposure was the sum of doses across all treatment cycles. SD Standard deviation, $U$ Unit 
Table 3 AbobotulinumtoxinA administration by muscle group and cycle

\begin{tabular}{llll}
\hline & Treatment Cycle 1 & Treatment Cycle 2 & Treatment Cycle 3 \\
\hline $\begin{array}{l}\text { Muscles injected, } \mathbf{n}(\%) \\
\text { Splenius capitis }\end{array}$ & $99(88.4)$ & & $\mathbf{N = 9 3}$ \\
Sternocleidomastoid & $75(67.0)$ & $87(89.7)$ & $84(90.3)$ \\
Trapezius & $73(65.2)$ & $67(69.1)$ & $63(67.7)$ \\
Levator scapulae & $67(59.8)$ & $66(68.0)$ & $62(66.7)$ \\
Scalenus (medius and anterior) & $29(25.9)$ & $60(61.9)$ & $57(61.3)$ \\
Semispinalis capitis & $38(33.9)$ & $31(32.0)$ & $28(30.1)$ \\
Longissimus & $23(20.5)$ & $39(40.2)$ & $25(37.6)$ \\
Other & $29(25.9)$ & $24(24.7)$ & $21(22.6)$ \\
\hline
\end{tabular}

patients. Most TEAEs $(n=156 / 220 ; 70.9 \%)$ were not considered by the investigators to be treatment related.

Seven patients reported eight serious TEAEs. There were two episodes of osteoarthritis (severe) and one each of: appendicitis, cholecystitis, dysphagia (severe), ischemic colitis (severe), thoracic vertebral fracture, and transient ischemic attack (severe). One serious TEAE (dysphagia), which occurred during treatment cycle 1, three days after receiving the first dose of $500 \mathrm{U} / 2-\mathrm{mL}$ dilution of abobotulinumtoxinA, was judged to be severe and treatment related. The injections had been made into the right splenius capitis muscle, left splenius capitis, sternocleidomastoid, levator scapulae and trapezius muscles. This event occurred in a 56-year-old, white female who had torticollis, laterocollis, and a history of dysphagia at enrollment to the lead-in study. The patient presented with great difficulty with swallowing and was unable to eat or drink. During an esophagogastroduodenoscopy 5 days later, a small Schatzki's ring in the distal esophagus was found. Although the small Schatzki ring could have been partly responsible for the presented symptoms, it was reported that the event may have been

Table 4 Summary of safety and tolerability across all treatment cycles

\begin{tabular}{ll}
\hline Patients, $n(\%)$ & Patients ( $N=112)$ \\
\hline Any TEAE Intensity & $70(62.5 \%)$ \\
Severe & $10(8.9 \%)$ \\
Moderate & $38(33.9 \%)$ \\
Mild & $53(47.3 \%)$ \\
Serious adverse events (SAEs) & $7(6.3 \%)$ \\
Treatment-related SAE & $33(29.5 \%)$ \\
Not related & $56(50.0 \%)$ \\
Missing & $1(0.9 \%)$ \\
Leading to study withdrawal & 0 \\
Leading to death & 0 \\
\hline
\end{tabular}

TEAE Treatment-emergent adverse event an underlying condition that was brought to light due to study drug administration. This event lasted from August 21st to October 1st, and the patient received the next dose of abobotulinumtoxinA on November 19th. Other reported serious TEAEs were considered not to be treatment related. None of the TEAEs led to study withdrawal or death. All patients recovered; one patient had sequelae following the severe, but not-related, event of a transient ischemic attack (TIA) that occurred during treatment cycle 1 .

\section{Efficacy}

The mean TWSTRS total score was 42.2 (SD 10.3) at baseline in the lead-in study (before first abobotulinumtoxinA administration). In the OLE study, the mean baseline (cycle 1, day 1) TWSTRS total score was 37.7 (SD 13.6) and declined to 30.1 (SD 12.8) at the cycle 3, week 12 visit. Thus, the mean change from the lead-in baseline was -11.7 (SD 11.2) at cycle 3, week 12 (Fig. 2). For each cycle, TWSTRS total and subscale scores decreased from day 1 to week 4 and increased between week 4 and week 12, though the week 12 scores remained lower than day 1 scores (Figs. 2 and 3). The mean TWSTRS scores at weeks 4 and 12 appeared to decrease for each successive cycle, with no return to baseline (Figs. 2 and 3).

\section{Discussion}

This study demonstrated that abobotulinumtoxinA injected at approved doses using a 2-mL dilution was safe and well tolerated while providing sustained improvements within and across treatment cycles in patients with CD. Most TEAEs reported were mild to moderate in severity and most were considered unrelated to treatment. Dysphagia, a common treatmentrelated side effect of all available botulinum toxin treatments for CD [9], occurred in $10.7 \%$ of patients treated in this study, slightly less than the $15 \%$ incidence reported in abobotulinumtoxinA clinical trials [10]. We 


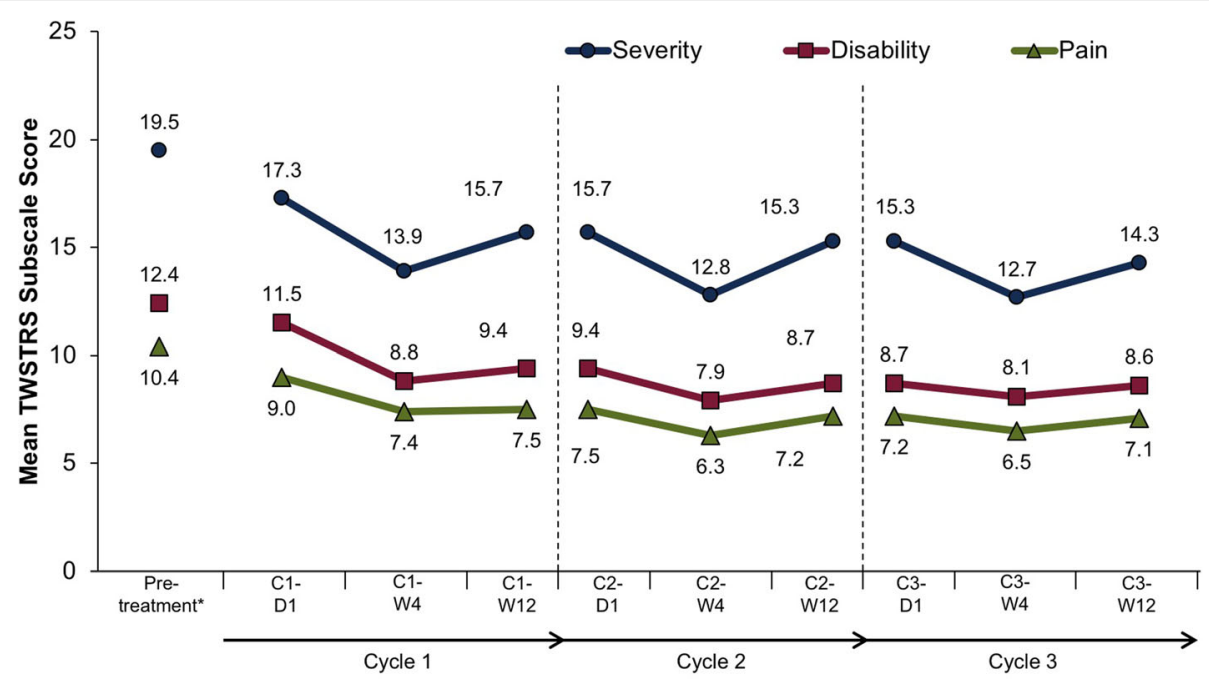

Fig. 3 Change from baseline in TWSTRS subscale scores. C, cycle; D, day; W, week. *Before first abobotulinumtoxinA administration

speculate that this may be attributable to the fact that the majority of patients enrolled in the OLE (77/112) had previously been treated with toxin during the leadin study, and some may have reached treatment "optimization" during the OLE. A recently published single-site retrospective cohort study [11] in which patients receiving optimized treatment for $C D$ reported lower rates of dysphagia supports this hypothesis. In that study the rates of dysphagia were also unrelated to total dose or dosing of the sternocleidomastoid muscle [11]. In the present study, dysphagia occurring in one patient $(<1 \%)$ with a history of dysphagia was considered to be a serious treatment-related adverse event, consistent with the incidence of severe dysphagia reported in $<5 \%$ of cases [9]. There were no discontinuations due to TEAEs. No new safety signals were detected in individuals treated for up to four cycles.

Consistent with the abobotulinumtoxinA pharmacological effect, 4-week efficacy was numerically greater than 12-week efficacy for each cycle. Results were consistent with the lead-in double-blind study and showed that abobotulinumtoxinA using a 2-mL dilution remained effective and well tolerated over four treatment cycles. Moreover, safety and efficacy with abobotulinumtoxinA using the $2-\mathrm{mL}$ dilution were similar to those with $1-\mathrm{mL}$ dilution.

\section{Conclusions}

Extended treatment of cervical dystonia with abobotulinumtoxinA over multiple treatment cycles using a 2-mL dilution is effective and has a positive risk-benefit profile while providing greater dosing flexibility to meet individual patient needs.

\section{Abbreviations}

BMI: Body mass index; C: Cycle; CD: Cervical dystonia; D: Day; OLE: Openlabel extension; FDA: U.S. Food and Drug Administration; ICH: International Conference on Harmonisation; SAE: Serious adverse event; SD: Standard deviation; TEAE: Treatment-emergent adverse event; TWSTRS: Toronto Western Spasmodic Torticollis Rating Scale; U: Unit; USA: United States of America; W: Week

\section{Acknowledgements}

The authors thank all patients involved in the study, as well as investigators and research staff in participating institutions, and Jim Otto, for his substantial contributions to the study while he was employed at Ipsen. The authors also thank Nicole Coolbaugh, CMPP, Kate Katsaval, BS, and Philip Sjostedy, BPharm, of The Medicine Group, LLC (New Hope, PA, USA) for providing medical writing support, which was sponsored by Ipsen (Cambridge, MA, USA) in accordance with Good Publication Practice guidelines.

\section{Authors' contributions}

Khashayar Dashtipour, Stefan Wietek, Bruce Rubin, Laxman Bahroo, and Richard Trosch: Authors contributed to conceptualization (formulation or evolution of research goals and aims), investigation (conducting the research), methodology (contributing to the development of the design and review of analysis), and writing (contributing to the original draft preparation, review, and editing; approval of final version). Pascal Maisonobe: Author contributed to formal analysis. The author(s) read and approved the final manuscript.

\section{Funding}

Ipsen, Cambridge, MA, USA.

\section{Availability of data and materials}

Where patient data can be anonymised, Ipsen will share all individual participant data that underlie the results reported in this article with qualified researchers who provide a valid research question. Study documents, such as the study protocol and clinical study report, are not always available. Proposals should be submitted to DataSharing@lpsen.com and will be assessed by a scientific review board. Data are available beginning 6 months and ending 5 years after publication; after this time, only raw data may be available.

\section{Ethics approval and consent to participate}

This study obtained appropriate institutional review board approval and was conducted under the provisions of the Declaration of Helsinki. Patients 
provided informed consent prior to their enrollment. This study was registered with ClinicalTrials.gov (identifier: NCT01753336).

\section{Consent for publication}

Not applicable.

\section{Competing interests}

KD: Advisor/consultant, and/or speaker: Abbvie, ACADIA, Acorda, Adamas, Allergan, Amneal, Impax, Ipsen, Lundbeck, Neurocrine, Revance, Sunovion, Teva, US WorldMeds; SW: Employment: Ipsen; BR: Employment (former): Ipsen; PM: Employment: Ipsen; LB: Speaker's bureaus: Allergan, Ipsen, Us WorldMeds; Consultant: US WorldMeds; RT: Speaker and/or Consultant: Ipsen, Neurocrine, Acadia, Amneal, and US WorldMeds.

\section{Author details}

'Loma Linda University School of Medicine, Loma Linda, CA 92350, USA. ${ }^{2}$ Ipsen, Cambridge, MA 02142, USA. ${ }^{3}$ Formerly of Ipsen, Cambridge, MA 02142, USA. ${ }^{4}$ Ipsen Pharma, 92100 Boulogne-Billancourt, France. ${ }^{5}$ Georgetown University Hospital, Washington, DC 20007, USA. ${ }^{6}$ Parkinson's and Movement Disorders Center, Farmington Hills, MI 48334, USA.

Received: 29 April 2020 Accepted: 10 August 2020

Published online: 31 August 2020

\section{References}

1. Dashtipour K, Lew M. Cervical dystonia. In: Stacy MA, editor. Handbook of Dystonia. London: Informa Healthcare; 2012. p. 144-58.

2. Defazio G, Jankovic J, Giel JL, Papapetropoulos S. Descriptive epidemiology of cervical dystonia. Tremor Other Hyperkinet Mov (N Y). 2013;3:tre-03-1934374-4372.

3. Marras C, Van den Eeden SK, Fross RD, Benedict-Albers KS, Klingman J, Leimpeter AD, Nelson LM, Risch N, Karter AJ, Bernstein AL, Tanner CM. Minimum incidence of primary cervical dystonia in a multiethnic health care population. Neurology. 2007;69:676-80.

4. Truong D, Duane DD, Jankovic J, Singer C, Seeberger LC, Comella CL, Lew MF, Rodnitzky RL, Danisi FO, Sutton JP, et al. Efficacy and safety of botulinum type a toxin (Dysport) in cervical dystonia: results of the first US randomized, double-blind, placebo-controlled study. Mov Disord. 2005;20:783-91.

5. Truong D, Brodsky M, Lew M, Brashear A, Jankovic J, Molho E, Orlova O, Timerbaeva S. Long-term efficacy and safety of botulinum toxin type a (Dysport) in cervical dystonia. Parkinsonism Relat Disord. 2010;16:316-23.

6. Hauser RA, Truong D, Hubble J, Coleman C, Beffy JL, Chang S, Picaut P. AbobotulinumtoxinA (Dysport) dosing in cervical dystonia: an exploratory analysis of two large open-label extension studies. J Neural Transm (Vienna). 2013;120:299-307.

7. Lew MF, Brashear A, Dashtipour K, Isaacson S, Hauser RA, Maisonobe P, Snyder D, Ondo W. A $500 \mathrm{U} / 2 \mathrm{~mL}$ dilution of abobotulinumtoxinA vs. placebo: randomized study in cervical dystonia. Int J Neurosci. 2018:128:619-26.

8. Consky E, Lang A. Clinical assessments of patients with cervical dystonia. In: Jankovic J, editor. Therapy with Botulinum Toxin. New York: Taylor \& Francis; 1994.

9. Contarino MF, Van Den Dool J, Balash Y, Bhatia K, Giladi N, Koelman JH, Lokkegaard A, Marti MJ, Postma M, Relja M, et al. Clinical practice: evidencebased recommendations for the treatment of cervical dystonia with botulinum toxin. Front Neurol. 2017;8:35.

10. Dysport [package insert]. Cambridge: Ipsen Biopharmaceuticals, Inc.; 2020.

11. Kutschenko A, Klietz M, Paracka L, Kollewe K, Schulte-Sutum A, Janssen T, Schrader C, Wegner F, Dressler D. Dysphagia in cervical dystonia patients receiving optimised botulinum toxin therapy: a single-center retrospective cohort study. J Neural Transm (Vienna). 2020;127(8):1161-5.

\section{Publisher's Note}

Springer Nature remains neutral with regard to jurisdictional claims in published maps and institutional affiliations.

Ready to submit your research? Choose BMC and benefit from:
- fast, convenient online submission
- thorough peer review by experienced researchers in your field
- rapid publication on acceptance
- support for research data, including large and complex data types
- gold Open Access which fosters wider collaboration and increased citations
- maximum visibility for your research: over 100M website views per year
At BMC, research is always in progress.
Learn more biomedcentral.com/submissions

